1 Hacettepe Journal of Mathematics and Statistics

$\bigcap$ Volume 45 (6) (2016), 1705-1718

\title{
Eigenvalue distribution of relaxed mixed constraint preconditioner for saddle point problems
}

\author{
Cui-Xia Li and Shi-Liang $\mathrm{Wu}^{*}$
}

\begin{abstract}
In this paper, the eigenvalue distribution of a family of relaxed mixed constraint preconditioner (RMCP) for the generalized saddle point problems is discussed in detail. Most of the bounds developed improve those appeared in previously published work.
\end{abstract}

Keywords: saddle point problems; inexact constraint preconditioner; eigenvalue 2000 AMS Classification: 65F10

Received: 07.09.2015 Accepted: 02.02.2016 Doi : 10.15672/HJMS.20164515686

\section{Introduction}

Consider the large, sparse and nonsingular linear system in saddle point form as

$$
\mathcal{A} x \equiv\left[\begin{array}{cc}
A & B^{T} \\
B & -C
\end{array}\right]\left[\begin{array}{l}
u \\
p
\end{array}\right]=\left[\begin{array}{l}
f \\
g
\end{array}\right] \equiv b,
$$

where $A \in \mathbb{R}^{n \times n}$ is symmetric positive definite (SPD), $B \in \mathbb{R}^{m \times n}$ with $m \leq n$ (possibly $m \ll n)$ is of full rank and $C \in \mathbb{R}^{m \times m}$ is symmetric semi-positive definite. Systems of the form (1.1) arise in a variety of scientific and engineering applications, such as constrained optimization, least squares and Stokes problems. We refer the reader to [10] for a more detailed list of applications and numerical solution techniques of (1.1).

In recent years, considerable effort has been invested in developing efficient solvers for systems of form (1.1). Recent works on sparse direct methods for symmetric saddle point problems have been developed, such as direct solver package [18] and $L D L^{T}$ factorization technique [19]. In fact, the memory and the computational requirements for solving saddle point problems (1.1) may seriously challenge the most efficient direct solution method available today. In actual implements, many iterative methods have to be recommended to solve saddle point problems (1.1), such as generalized successive overrelaxation (GSOR) method [2], modified SSOR (MSSOR) method [33], Hermitian and skew-Hermitian splitting method $[3-7,11,12]$ and so on. However, well established

${ }^{*}$ School of Mathematics and Statistics, Anyang Normal University, Anyang, 455000, China Corresponding author: wushiliang1999@126.com or slwu@aynu.edu.cn 
iterative methods such as Krylov subspace methods are very slow or even fail to converge if not conveniently preconditioned, it follows that preconditioning technique is a key ingredient for the success of Krylov subspace methods in applications. Most of the recent work on saddle point problems has focused on the development of preconditioners for Krylov subspace methods, especially block preconditioners and multilevel schemes. We refer the reader to [10] for a comprehensive survey of existing approaches for solving saddle point problems.

An important class of preconditioners is based on the block LU factorization of the coefficient matrix $\mathcal{A}[8,9]$. This class includes a variety of block diagonal and block triangular preconditioners [8,9,20-26, 28-30,39-43]. Based on the Hermitian and skewHermitian splitting of the coefficient matrix $\mathcal{A}$, the HSS preconditioner is established $[3-6,12]$. Based on the Dimensional Splitting (DS) of the coefficient matrix $\mathcal{A}$, a relaxed dimensional factorization preconditioner for Navier-Stokes equations is proposed [13,14]. Based on the augmented Lagrangian (AL) reformulation of the saddle point problem, AL-type preconditioners appear to be remarkably robust for a broad range of problem parameters, and they are currently the focus of intense development in $[15,16]$.

As is known to all, the major issue of preconditioning technique is to find a good approximation of the inverse of the coefficient matrix $\mathcal{A}$. To accelerate Krylov solvers for saddle point problems, constraint preconditioner is another type of preconditioning techniques and has been first introduced in constrained optimization for $C=0$ [31]. It has been proved [31] that the eigenvalues of the preconditioned matrix are all real and positive. The strategy of constraint preconditioner is that a suitable approximation of the $(1,1)$ block $A$ instead of the $(1,1)$ block $A$ leads to a good approximation of the inverse of the coefficient matrix $\mathcal{A}$. Dollar [32] has extended these results in [31] by allowing the $(2,2)$ block to be symmetric and positive semidefinite. Further, the general symmetric $(2,2)$ block has been discussed [1] and the nonsymmetric $(1,1)$ block has been discussed [27]. Constraint preconditioner can be written as the inverse of a matrix whose non diagonal blocks are the same as those in $\mathcal{A}$, but their application may be very costly since it requires the solution of a linear system at each iteration with an appropriate Schur complement $S$ as the coefficient matrix $\mathcal{A}$. A computationally efficient inexact constraint preconditioner (ICP) is represented by an approximation of $S$ (or of $S^{-1}$ ) by means of an incomplete Cholesky factorization or a sparse approximate inverse. The application of ICP is cheaper with respect to the constraint preconditioner. An exhaustive analysis of spectral properties of ICP together with development of eigenvalue bounds are performed in [36]. ICP has been proved much more robust and performing than ILU preconditioners with variable fill-in, computed on the whole saddle point matrix from a number of realistic coupled consolidation problems [38].

Recently, drawing on the previous works: [34-36], Bergamaschi and Martínez [37] discussed a family of relaxed mixed constraint preconditioner (RMCP) as follows:

$$
\mathcal{M}_{\omega}=\left[\begin{array}{cc}
I & 0 \\
B P_{A}^{-1} & I
\end{array}\right]\left[\begin{array}{cc}
P_{A} & 0 \\
0 & -\omega P_{S}
\end{array}\right]\left[\begin{array}{cc}
I & P_{A}^{-1} B^{T} \\
0 & I
\end{array}\right]=\left[\begin{array}{cc}
P_{A} & B^{T} \\
B & B P_{A}^{-1} B^{T}-\omega P_{S}
\end{array}\right],
$$

where $\omega$ is a real acceleration parameter, $P_{A}$ is a suitable approximation of the $(1,1)$ block $A$ and $P_{S}$ is a suitable approximation of the Schur complement matrix $S=B P_{A}^{-1} B^{T}+$ $C$. A detailed spectral analysis of RMCP was presented in [37]. In this paper, we focus on the relaxed mixed constraint preconditioner (RMCP) for symmetric saddle point problems (1.1). The spectral properties of the preconditioned matrix are given and some corresponding presented results in $[36,37,44]$ are improved.

The paper is organized as follows. In Section 2, the spectral distribution of a class of the parameterized saddle point problems is characterized, which extends the corresponding theoretical results in $[17,36]$. In Section 3, we discuss the eigenvalue distribution of 
$\mathcal{M}_{\omega}^{-1} \mathcal{A}$ in detail and promote some corresponding presented results in [36,37, 44]. The conclusions are drawn in Section 4.

\section{Eigenvalues of $\mathcal{A}_{\omega}$}

To make the spectral analysis of $\mathcal{M}_{\omega}^{-1} \mathcal{A}$ easily, the spectral distribution of a class of the parameterized saddle point matrix is characterized.

Given that $A \in \mathbb{R}^{n \times n}$ is symmetric positive definite (SPD), $B \in \mathbb{R}^{m \times n}(m \leq n)$ is of full rank and $C \in \mathbb{R}^{m \times m}$ is symmetric positive definite. For $\omega>0$, we are interested in the eigenvalues of

$$
\mathcal{A}_{\omega} u \equiv\left[\begin{array}{cc}
A & \frac{1}{\sqrt{\omega}} B^{T} \\
-\frac{1}{\sqrt{\omega}} B & \frac{1}{\omega} C
\end{array}\right]\left[\begin{array}{l}
u_{1} \\
u_{2}
\end{array}\right]=\lambda\left[\begin{array}{l}
u_{1} \\
u_{2}
\end{array}\right] \equiv \lambda u
$$

or

$$
\begin{aligned}
A u_{1}+\frac{1}{\sqrt{\omega}} B^{T} u_{2} & =\lambda u_{1}, \\
-\frac{1}{\sqrt{\omega}} B u_{1}+\frac{1}{\omega} C u_{2} & =\lambda u_{2} .
\end{aligned}
$$

For the purposes of our discussion, the following notation regarding the eigenvalues of SPD matrices $A, B B^{T}$ and $C$ are required:

$$
\begin{array}{ll}
0<\alpha_{A}=\lambda_{\min }(A), & \beta_{A}=\lambda_{\max }(A), \\
0 \leq \alpha_{S}=\lambda_{\min }\left(B B^{T}\right), & \beta_{S}=\lambda_{\max }\left(B B^{T}\right), \\
0<\alpha_{C}=\lambda_{\min }(C), & \beta_{C}=\lambda_{\max }(C) .
\end{array}
$$

Obviously, matrix $\mathcal{A}_{\omega}$ has at most $n-m$ eigenvalues satisfying

$$
\alpha_{A} \leq \lambda \leq \beta_{A}
$$

with eigenvectors $u=\left(u_{1}^{T}, 0\right)^{T}$ and $B u_{1}=0$. One can see for instance Proposition 2.2 in [17].

Throughout this section, we define, for some $s, u_{2} \neq 0$,

$$
\eta_{A}=\frac{s^{T} A s}{s^{T} s} \in\left[\alpha_{A}, \beta_{A}\right], \eta_{C}=\frac{u_{2}^{T} C u_{2}}{u_{2}^{T} u_{2}} \in\left[\alpha_{C}, \beta_{C}\right], \eta_{S}=\frac{u_{2}^{T} B B^{T} u_{2}}{u_{2}^{T} u_{2}} \in\left[\alpha_{S}, \beta_{S}\right] .
$$

The proof of Theorem 2.2 is based on the following Lemma 2.1, which is from [36].

2.1. Lemma. [36] Let $\lambda \notin\left[\alpha_{A}, \beta_{A}\right]$. Then, for every $z \neq 0$, there exists a vector $s \neq 0$ such that

$$
\frac{z^{T}(A-\lambda I)^{-1} z}{z^{T} z}=\left(\frac{s^{T} A s}{s^{T} s}-\lambda\right)^{-1}=\left(\eta_{A}-\lambda\right)^{-1} .
$$

2.2. Theorem. The real eigenvalues of Equation (2.1) not lying in $\left[\alpha_{A}, \beta_{A}\right]$ satisfy

$$
\frac{1}{\omega}\left(\alpha_{C}+\frac{\alpha_{S}}{\beta_{A}}\right) \leq \frac{1}{\omega}\left(\eta_{C}+\frac{\eta_{S}}{\eta_{A}}\right) \leq \lambda \leq \max \left\{\eta_{A}, \frac{1}{\omega} \eta_{C}\right\} \leq \max \left\{\beta_{A}, \frac{1}{\omega} \beta_{C}\right\} .
$$

Proof. Let $\lambda \in \mathbb{R}$ with $\lambda \notin\left[\alpha_{A}, \beta_{A}\right]$ and let $u$ such that $B u_{1} \neq 0$ and $B^{T} u_{2} \neq 0$. Since $A-\lambda I$ is invertible, from (2.2) we have

$$
u_{1}=-\frac{1}{\sqrt{\omega}}(A-\lambda I)^{-1} B^{T} u_{2}
$$

Substituting (2.4) into (2.3) yields

$$
\frac{1}{\omega} B(A-\lambda I)^{-1} B^{T} u_{2}+\frac{1}{\omega} C u_{2}-\lambda u_{2}=0 .
$$


Premultiplying (2.5) by $\frac{u_{2}^{T}}{u_{2}^{T} u_{2}}$ leads to

$$
\frac{1}{\omega} \frac{u_{2}^{T} B(A-\lambda I)^{-1} B^{T} u_{2}}{u_{2}^{T} u_{2}}+\frac{1}{\omega} \eta_{C}-\lambda=0 .
$$

Based on Lemma 2.1, from (2.6) we have

or,

$$
\frac{1}{\omega}\left(\eta_{A}-\lambda\right)^{-1} \eta_{S}+\frac{1}{\omega} \eta_{C}-\lambda=0 .
$$

$$
\omega \lambda^{2}-\left(\eta_{C}+\omega \eta_{A}\right) \lambda+\eta_{S}+\eta_{A} \eta_{C}=0 .
$$

The lager solution of (2.7) is

$$
\begin{aligned}
\lambda_{2} & =\frac{\eta_{C}+\omega \eta_{A}+\sqrt{\left(\eta_{C}+\omega \eta_{A}\right)^{2}-4 \omega\left(\eta_{S}+\eta_{A} \eta_{C}\right)}}{2 \omega} \\
& =\frac{\eta_{C}+\omega \eta_{A}+\sqrt{\left(\eta_{C}-\omega \eta_{A}\right)^{2}-4 \omega \eta_{S}}}{2 \omega} \\
& \leq \max \left\{\eta_{A}, \frac{1}{\omega} \eta_{C}\right\} .
\end{aligned}
$$

The smaller solution of (2.7) is

$$
\begin{aligned}
\lambda_{1} & =\frac{\eta_{C}+\omega \eta_{A}-\sqrt{\left(\eta_{C}+\omega \eta_{A}\right)^{2}-4 \omega\left(\eta_{S}+\eta_{A} \eta_{C}\right)}}{2 \omega} \\
& =\frac{2\left(\eta_{S}+\eta_{A} \eta_{C}\right)}{\eta_{C}+\omega \eta_{A}+\sqrt{\left(\eta_{C}-\omega \eta_{A}\right)^{2}-4 \omega \eta_{S}}} \\
& \geq \frac{\eta_{S}+\eta_{A} \eta_{C}}{\max \left\{\omega \eta_{A}, \eta_{C}\right\}}=\frac{1}{\omega}\left(\eta_{C}+\frac{\eta_{S}}{\eta_{A}}\right) .
\end{aligned}
$$

The last equation follows from the inequality $\eta_{C}<\omega \eta_{A}$ (otherwise we would have $\lambda_{1}>$ $\eta_{A}>\alpha_{A}$ against the assumption). Hence,

$$
\lambda_{1} \geq \frac{1}{\omega}\left(\eta_{C}+\frac{\eta_{S}}{\eta_{A}}\right) \geq \frac{1}{\omega}\left(\alpha_{C}+\frac{\alpha_{S}}{\beta_{A}}\right) .
$$

2.1. Corollary. The real eigenvalues of Equation (2.1) satisfy

$$
\min \left\{\alpha_{A}, \frac{1}{\omega}\left(\alpha_{C}+\frac{\alpha_{S}}{\beta_{A}}\right)\right\} \leq \lambda \leq \max \left\{\beta_{A}, \frac{1}{\omega} \beta_{C}\right\}
$$

In the sequel, we will denote any complex eigenvalue as

$$
\lambda=\lambda_{R}+i \lambda_{I}
$$

2.2. Corollary. The complex eigenvalues of Equation (2.1) satisfy

$$
\frac{\omega \alpha_{A}+\alpha_{C}}{2 \omega} \leq \lambda_{R} \leq \frac{\omega \beta_{A}+\beta_{C}}{2 \omega},\left|\lambda_{I}\right| \leq \sqrt{\frac{\beta_{S}}{\omega}} .
$$

Proof. From (2.7), we have

$$
\begin{aligned}
\lambda_{R} & =\frac{\eta_{C}+\omega \eta_{A}}{2 \omega}, \\
\lambda_{R}^{2}+\lambda_{I}^{2} & =\frac{\eta_{S}+\eta_{A} \eta_{C}}{\omega}
\end{aligned}
$$

By simple computations, from (2.8) we have

$$
\frac{\omega \alpha_{A}+\alpha_{C}}{2 \omega} \leq \lambda_{R} \leq \frac{\omega \beta_{A}+\beta_{C}}{2 \omega} .
$$


Combining (2.8) and (2.9), we have

$$
\begin{aligned}
\left|\lambda_{I}\right| & =\sqrt{\frac{\eta_{S}+\eta_{A} \eta_{C}}{\omega}-\left(\frac{\eta_{C}+\omega \eta_{A}}{2 \omega}\right)^{2}} \\
& =\sqrt{\frac{4 \omega\left(\eta_{S}+\eta_{A} \eta_{C}\right)-\left(\eta_{C}+\omega \eta_{A}\right)^{2}}{4 \omega^{2}}} \\
& =\sqrt{\frac{\eta_{S}}{\omega}-\frac{\left(\eta_{C}-\omega \eta_{A}\right)^{2}}{4 \omega^{2}}} \leq \sqrt{\frac{\eta_{S}}{\omega}} \leq \sqrt{\frac{\beta_{S}}{\omega}} .
\end{aligned}
$$

Remark 2.1 When $\omega=1$, Theorem 2.2 reduces to Theorem 1 [36], Corollary 2.1 reduces to Corollary 1 [36] and Corollary 2.2 reduces to Proposition 1 [36]. Specifically, this result in Corollary 2.1 with $\omega=1$ improves that of Proposition 2.12 in [17], which provides a lower bound for $\lambda \geq \min \left\{\alpha_{A}, \alpha_{C}\right\}$.

Example 2.1

$$
\mathcal{A}_{\omega}=\left[\begin{array}{ccc}
\beta_{A} & 0 & \frac{1}{\sqrt{\omega}} \times 1 \\
0 & \alpha_{A} & \frac{1}{\sqrt{\omega}} \times 1 \\
-\frac{1}{\sqrt{\omega}} \times 1 & -\frac{1}{\sqrt{\omega}} \times 1 & \frac{1}{\omega} \times c
\end{array}\right], \alpha_{S}=\beta_{S}=2, \omega=4 .
$$

If $\beta_{A}=3, \alpha_{A}=2.9$ and $c=1$, the eigenvalues of $\mathcal{A}_{\omega}$ are $\lambda\left(\mathcal{A}_{\omega}\right)=\{0.4501,2.7372,2.9627\}$. Obviously, $\alpha_{C}=\beta_{C}=1$. From Corollary 2.1, we have

$$
0.4167<\lambda<3 \text {. }
$$

If $\beta_{A}=3, \alpha_{A}=2$ and $c=4$, the eigenvalues of $\mathcal{A}_{\omega}$ are $\lambda\left(\mathcal{A}_{\omega}\right)=\{2.8846,1.5577+$ $0.2949 i, 1.5577-0.2949 i\}$. Obviously, $\alpha_{C}=\beta_{C}=4$. From Corollary 2.1, we have

$$
\frac{7}{6}<\lambda<3 \text {. }
$$

From Corollary 2.2, we have

$$
\frac{3}{2}<\lambda_{R}<2,\left|\lambda_{I}\right| \leq \frac{\sqrt{2}}{2} .
$$

Numerical results show that Corollary 2.1 provides some valid bounds for all the real eigenvalues of $\mathcal{A}_{\omega}$ and Corollary 2.2 provides some valid bounds for all the complex eigenvalues of $\mathcal{A}_{\omega}$.

\section{Spectral analysis of $\mathcal{A} \mathcal{M}_{\omega}^{-1}$}

It is not difficult to find that the spectral of $\mathcal{M}_{\omega}^{-1} \mathcal{A}$ is equivalent to the spectral of $\mathcal{A} \mathcal{M}_{\omega}^{-1}$. Here we focus on the bounds for the eigenvalues of $\mathcal{A} \mathcal{M}_{\omega}^{-1}$ to obtain the bounds for the eigenvalues of $\mathcal{M}_{\omega}^{-1} \mathcal{A}$. Making this strategy to discuss the bounds for the eigenvalues of the corresponding preconditioned matrix, one can see $[24,25,36,40,41,43]$ for more details.

In fact, $\mathcal{A N}_{\omega}^{-1} z=\lambda z$ can be expressed as

$$
\mathcal{A} \nu=\lambda \mathcal{M}_{\omega} \nu, \nu=\mathcal{M}_{\omega}^{-1} z .
$$

To investigate the spectral properties of $\mathcal{M}_{\omega}^{-1} \mathcal{A}, P_{A}$ and $P_{S}$, respectively, are SPD approximations of $A$ and $S=B P_{A}^{-1} B^{T}+C$. $P_{A}^{-1}$ and $P_{S}^{-1}$ can also be viewed as preconditioners for the corresponding matrices, so that we can define the following SPD preconditioned matrices:

$$
\mathcal{P}=\left[\begin{array}{cc}
P_{A} & 0 \\
0 & P_{S}
\end{array}\right] \text { and } S_{P}=P_{S}^{-1 / 2} S P_{S}^{-1 / 2}
$$


1710

Since $\mathcal{P}$ is symmetric positive definite, the problem of finding the eigenvalues of $\mathcal{M}_{\omega}^{-1} \mathcal{A}$ with $u=\mathcal{P}_{+}^{\frac{1}{2}} \nu$ is equivalent to solving

$$
\mathcal{P}^{-\frac{1}{2}} \mathcal{A} \mathcal{P}^{-\frac{1}{2}} u=\lambda \mathcal{P}^{-\frac{1}{2}} \mathcal{M}_{\omega} \mathcal{P}^{-\frac{1}{2}} u \text {. }
$$

That is,

$$
\left[\begin{array}{cc}
A_{P} & R^{T} \\
R & -\widehat{C}
\end{array}\right]\left[\begin{array}{l}
u_{1} \\
u_{2}
\end{array}\right]=\lambda\left[\begin{array}{cc}
I & R^{T} \\
R & R R^{T}-\omega I
\end{array}\right]\left[\begin{array}{l}
u_{1} \\
u_{2}
\end{array}\right]
$$

where $R=P_{S}^{-1 / 2} B P_{A}^{-1 / 2}, A_{P}=P_{A}^{-1 / 2} A P_{A}^{-1 / 2}$ and $\widehat{C}=P_{S}^{-1 / 2} C P_{S}^{-1 / 2}$. Note that $R R^{T}=S_{P}-\widehat{C}$ and the inverse of the right side matrix product in (3.1) can be written as

$$
\begin{aligned}
{\left[\begin{array}{cc}
I & R^{T} \\
R & R R^{T}-\omega I
\end{array}\right]^{-1} } & =\left[\left[\begin{array}{cc}
I & 0 \\
R & -\sqrt{\omega} I
\end{array}\right]\left[\begin{array}{cc}
I & R^{T} \\
0 & \sqrt{\omega} I
\end{array}\right]\right]^{-1} \\
& =\left[\begin{array}{cc}
I & R^{T} \\
0 & \sqrt{\omega} I
\end{array}\right]^{-1}\left[\begin{array}{cc}
I & 0 \\
R & -\sqrt{\omega} I
\end{array}\right]^{-1} \\
& =\left[\begin{array}{cc}
I & -\frac{1}{\sqrt{\omega}} R^{T} \\
0 & \frac{1}{\sqrt{\omega}} I
\end{array}\right]\left[\begin{array}{cc}
I & 0 \\
\frac{1}{\sqrt{\omega}} R & -\frac{1}{\sqrt{\omega}} I
\end{array}\right] \\
& \equiv \mathcal{U L},
\end{aligned}
$$

so that the eigenvalues of (3.1) are the same as those of $\mathcal{L} \mathcal{P}^{-\frac{1}{2}} \mathcal{A P} \mathcal{P}^{-\frac{1}{2}} U_{x}=\lambda x$ which reads:

$$
\left[\begin{array}{cc}
A_{P} & \frac{1}{\sqrt{\omega}}\left(I-A_{P}\right) R^{T} \\
-\frac{1}{\sqrt{\omega}} R\left(I-A_{P}\right) & \frac{1}{\omega}\left(R\left(2 I-A_{P}\right) R^{T}+\widehat{C}\right)
\end{array}\right]\left[\begin{array}{l}
x_{1} \\
x_{2}
\end{array}\right]=\lambda\left[\begin{array}{l}
x_{1} \\
x_{2}
\end{array}\right] .
$$

Let us assume that

$$
\begin{aligned}
& 0<\alpha_{A}=\lambda_{\min }\left(A_{P}\right)<1<\lambda_{\max }\left(A_{P}\right)=\beta_{A}, \\
& 0<\alpha_{S}=\lambda_{\min }\left(S_{P}\right)<1<\lambda_{\max }\left(S_{P}\right)=\beta_{S}, \\
& 0 \leq \alpha_{C}=\lambda_{\min }(\widehat{C})<\lambda_{\max }(\widehat{C})=\beta_{C} .
\end{aligned}
$$

Obviously, the eigenvalues of the projected matrix $A_{R}=\left(R R^{T}\right)^{-1} R A_{P} R^{T}$ is also important in the spectral analysis of the preconditioned matrices. In $[36,37,44]$, it is shown that $\left[\alpha_{A}^{R}, \beta_{A}^{R}\right] \subset\left[\alpha_{A}, \beta_{A}\right]$, where $\alpha_{A}^{R}=\lambda_{\min }\left(A_{R}\right)$ and $\beta_{A}^{R}=\lambda_{\max }\left(A_{R}\right)$.

Throughout this section, we will use the following notation:

$$
\theta_{S}=\frac{x_{2}^{T} S_{P} x_{2}}{x_{2}^{T} x_{2}}, \theta_{A}^{R}=\frac{x_{2}^{T} R A_{P} R^{T} x_{2}}{x_{2}^{T} R R^{T} x_{2}}, \theta_{A}=\frac{s^{T} A_{P} s}{s^{T} s}, \theta_{C}=\frac{x_{2}^{T} \widehat{C} x_{2}}{x_{2}^{T} x_{2}},
$$

for some $s, x_{2} \neq 0$. It follows that $\theta_{A}^{R} \in\left[\alpha_{A}^{R}, \beta_{A}^{R}\right]$ and $\frac{x_{2}^{T} R R^{T} x_{2}}{x_{2}^{T} x_{2}}=\theta_{S}-\theta_{C} \geq 0$.

To obtain the bounds for the eigenvalues of $\mathcal{M}_{\omega}^{-1} \mathcal{A}$, we need the following lemma.

3.1. Lemma. Let $H=R\left(2 I-A_{P}\right) R^{T}+\widehat{C}, P=R\left(I-A_{P}\right)^{2} R^{T}$ and $\beta_{A}^{R}<2$. If $\alpha_{A}^{R}<1$, then

$$
\begin{aligned}
& \lambda(H) \in\left[\alpha_{S}\left(2-\beta_{A}^{R}\right)+\alpha_{C}\left(\beta_{A}^{R}-1\right), \beta_{S}\left(2-\alpha_{A}^{R}\right)-\alpha_{C}\left(1-\alpha_{A}^{R}\right)\right], \\
& \lambda(P) \leq\left(\beta_{S}-\alpha_{C}\right) \max \left\{\left(1-\alpha_{A}^{R}\right)^{2},\left(\beta_{A}^{R}-1\right)^{2}\right\} .
\end{aligned}
$$

If $\alpha_{A}^{R} \geq 1$, then

$$
\begin{aligned}
& \lambda(H) \in\left[\alpha_{S}\left(2-\beta_{A}^{R}\right)+\alpha_{C}\left(\beta_{A}^{R}-1\right), \beta_{S}\left(2-\alpha_{A}^{R}\right)+\beta_{C}\left(\alpha_{A}^{R}-1\right)\right], \\
& \lambda(P) \leq\left(\beta_{S}-\alpha_{C}\right) \max \left\{\left(1-\alpha_{A}^{R}\right)^{2},\left(\beta_{A}^{R}-1\right)^{2}\right\} .
\end{aligned}
$$


Proof. Based on the results in [36,44], here we only need prove that $\lambda(H) \leq \beta_{S}(2-$ $\left.\alpha_{A}^{R}\right)+\beta_{C}\left(\alpha_{A}^{R}-1\right)$ for $\alpha_{A}^{R} \geq 1$.

In fact, $\lambda(H) \in\left[\min q\left(x_{2}, H\right), \max q\left(x_{2}, H\right)\right]$, where

$$
\begin{aligned}
q\left(x_{2}, H\right) & =\frac{x_{2}^{T}\left(R\left(2 I-A_{P}\right) R^{T}+\widehat{C}\right) x_{2}}{x_{2}^{T} x_{2}} \\
& =\left(\theta_{S}-\theta_{C}\right)\left(2-\theta_{A}^{R}\right)+\theta_{C} .
\end{aligned}
$$

Because the function on the right hand side is decreasing in $\theta_{A}^{R}$, then

$$
\begin{aligned}
\max q\left(x_{2}, H\right) & \leq\left(\theta_{S}-\theta_{C}\right)\left(2-\alpha_{A}^{R}\right)+\theta_{C} \\
& =\theta_{S}\left(2-\alpha_{A}^{R}\right)+\theta_{C}\left(\alpha_{A}^{R}-1\right) \\
& \leq \beta_{S}\left(2-\alpha_{A}^{R}\right)+\beta_{C}\left(\alpha_{A}^{R}-1\right) .
\end{aligned}
$$

The proof is completed.

Investigating the results in Lemma $2[36,44]$, the bounds for the eigenvalues of $R(2 I-$ $\left.A_{P}\right) R^{T}+\widehat{C}$ and $R\left(I-A_{P}\right)^{2} R^{T}$ are provided just when $\beta_{A}^{R}<2$ and $\alpha_{A}^{R}<1$. In this case, it is easy to see that the results in Lemma 3.1 perfect the corresponding theoretical results in Lemma $2[36,44]$. Based on Lemma 3.1, it is easy to obtain the following results.

3.1. Corollary. Let $H=\frac{1}{\omega}\left(R\left(2 I-A_{P}\right) R^{T}+\widehat{C}\right), P=\frac{1}{\omega} R\left(I-A_{P}\right)^{2} R^{T}$ and $\beta_{A}^{R}<2$. If $\alpha_{A}^{R}<1$, then

$$
\begin{aligned}
& \lambda(H) \in\left[\frac{\alpha_{S}\left(2-\beta_{A}^{R}\right)+\alpha_{C}\left(\beta_{A}^{R}-1\right)}{\omega}, \frac{\beta_{S}\left(2-\alpha_{A}^{R}\right)-\alpha_{C}\left(1-\alpha_{A}^{R}\right)}{\omega}\right], \\
& \lambda(P) \leq \frac{\left(\beta_{S}-\alpha_{C}\right)}{\omega} \max \left\{\left(1-\alpha_{A}^{R}\right)^{2},\left(\beta_{A}^{R}-1\right)^{2}\right\} .
\end{aligned}
$$

If $\alpha_{A}^{R} \geq 1$, then

$$
\begin{aligned}
& \lambda(H) \in\left[\frac{\alpha_{S}\left(2-\beta_{A}^{R}\right)+\alpha_{C}\left(\beta_{A}^{R}-1\right)}{\omega}, \frac{\beta_{S}\left(2-\alpha_{A}^{R}\right)+\beta_{C}\left(\alpha_{A}^{R}-1\right)}{\omega}\right], \\
& \lambda(P) \leq \frac{\left(\beta_{S}-\alpha_{C}\right)}{\omega} \max \left\{\left(1-\alpha_{A}^{R}\right)^{2},\left(\beta_{A}^{R}-1\right)^{2}\right\} .
\end{aligned}
$$

Obviously, Corollary 3.1 is a generalization of Lemma 3.1. When $\omega=1$, Corollary 3.1 reduces to Lemma 3.1.

Based on Theorem 3 in [36,44] and Corollary 3.1, we have the following results.

3.2. Theorem. Let $\beta_{A}<2$.

For $\alpha_{A}^{R}<1$, the real eigenvalues of (3.2) satisfy

$$
\min \left\{\alpha_{A}, \frac{\alpha_{S}+\alpha_{C}\left(\beta_{A}-1\right)}{\omega \beta_{A}}\right\} \leq \lambda \leq \max \left\{\beta_{A}, \frac{\beta_{S}\left(2-\alpha_{A}^{R}\right)-\alpha_{C}\left(1-\alpha_{A}^{R}\right)}{\omega}\right\} .
$$

And if $\lambda_{I} \neq 0$, then the complex eigenvalues of (3.2) satisfy

$$
\begin{array}{r}
\frac{\omega \alpha_{A}+\alpha_{S}\left(2-\beta_{A}^{R}\right)+\alpha_{C}\left(\beta_{A}^{R}-1\right)}{2 \omega} \leq \lambda_{R} \leq \frac{\omega \beta_{A}+\beta_{S}\left(2-\alpha_{A}^{R}\right)-\alpha_{C}\left(1-\alpha_{A}^{R}\right)}{2 \omega}, \\
\left|\lambda_{I}\right| \leq \sqrt{\frac{\beta_{S}-\alpha_{C}}{\omega}} \max \left\{1-\alpha_{A}^{R},\left|\beta_{A}^{R}-1\right|\right\} .
\end{array}
$$

For $\alpha_{A}^{R} \geq 1$, the real eigenvalues of (3.2) satisfy

$$
\min \left\{\alpha_{A}, \frac{\alpha_{S}+\alpha_{C}\left(\beta_{A}-1\right)}{\omega \beta_{A}}\right\} \leq \lambda \leq \max \left\{\beta_{A}, \frac{\beta_{S}\left(2-\alpha_{A}^{R}\right)+\beta_{C}\left(\alpha_{A}^{R}-1\right)}{\omega}\right\} .
$$


And if $\lambda_{I} \neq 0$, then the complex eigenvalues of (3.2) satisfy

$$
\begin{array}{r}
\frac{\omega \alpha_{A}+\alpha_{S}\left(2-\beta_{A}^{R}\right)+\alpha_{C}\left(\beta_{A}^{R}-1\right)}{2 \omega} \leq \lambda_{R} \leq \frac{\omega \beta_{A}+\beta_{S}\left(2-\alpha_{A}^{R}\right)+\beta_{C}\left(\alpha_{A}^{R}-1\right)}{2 \omega}, \\
\left|\lambda_{I}\right| \leq \sqrt{\frac{\beta_{S}-\alpha_{C}}{\omega}} \max \left\{\alpha_{A}^{R}-1,\left|\beta_{A}^{R}-1\right|\right\} .
\end{array}
$$

Proof. The proof is similar to the proof of Theorem 3 in [36]. One can see [36] for more details.

Obviously, when $\omega=1$, the following results are obtained.

3.2. Corollary. Let $\beta_{A}<2$.

For $\alpha_{A}^{R}<1$, the real eigenvalues of (3.2) satisfy

$$
\min \left\{\alpha_{A}, \frac{\alpha_{S}}{\beta_{A}}+\frac{\alpha_{C}\left(\beta_{A}-1\right)}{\beta_{A}}\right\} \leq \lambda \leq \max \left\{\beta_{A}, \beta_{S}\left(2-\alpha_{A}^{R}\right)-\alpha_{C}\left(1-\alpha_{A}^{R}\right)\right\} .
$$

And if $\lambda_{I} \neq 0$, then the complex eigenvalues of (3.2) satisfy

$$
\begin{gathered}
\frac{\alpha_{A}+\alpha_{S}\left(2-\beta_{A}^{R}\right)+\alpha_{C}\left(\beta_{A}^{R}-1\right)}{2} \leq \lambda_{R} \leq \frac{\beta_{A}+\beta_{S}\left(2-\alpha_{A}^{R}\right)-\alpha_{C}\left(1-\alpha_{A}^{R}\right)}{2}, \\
\left|\lambda_{I}\right| \leq \sqrt{\beta_{S}-\alpha_{C}}\left(\beta_{A}^{R}-1\right) .
\end{gathered}
$$

For $\alpha_{A}^{R} \geq 1$, the real eigenvalues of (3.2) satisfy

$$
\min \left\{\alpha_{A}, \frac{\alpha_{S}}{\beta_{A}}+\frac{\alpha_{C}\left(\beta_{A}-1\right)}{\beta_{A}}\right\} \leq \lambda \leq \max \left\{\beta_{A}, \beta_{S}\left(2-\alpha_{A}^{R}\right)+\beta_{C}\left(\alpha_{A}^{R}-1\right)\right\} .
$$

And if $\lambda_{I} \neq 0$, then the complex eigenvalues of (3.2) satisfy

$$
\begin{gathered}
\frac{\alpha_{A}+\alpha_{S}\left(2-\beta_{A}^{R}\right)+\alpha_{C}\left(\beta_{A}^{R}-1\right)}{2} \leq \lambda_{R} \leq \frac{\beta_{A}+\beta_{S}\left(2-\alpha_{A}^{R}\right)+\beta_{C}\left(\alpha_{A}^{R}-1\right)}{2}, \\
\left|\lambda_{I}\right| \leq \sqrt{\beta_{S}-\alpha_{C}}\left(\beta_{A}^{R}-1\right) .
\end{gathered}
$$

Remark 3.1 From Corollary 3.2, we know that for $\alpha_{A}^{R}<1$ and $\lambda_{I} \neq 0$, the upper bound of $\lambda_{R}$ is sharper than the upper bound of $\lambda_{R}$ in $[36,44]$. In fact, one can easily see the following result, that is,

$$
0<\frac{\beta_{A}+\beta_{S}\left(2-\alpha_{A}^{R}\right)-\alpha_{C}\left(1-\alpha_{A}^{R}\right)}{2} \leq \frac{\beta_{A}+\beta_{S}\left(2-\alpha_{A}^{R}\right)+\alpha_{C}\left(1-\alpha_{A}^{R}\right)}{2} .
$$

If $C \equiv 0$, then $\widehat{C}=P_{S}^{-1 / 2} C P_{S}^{-1 / 2}=0$. It follows that $\alpha_{C}=\beta_{C}=0$. Then the bounds of Theorem 3.2 simplify is stated in the following.

3.3. Corollary. Let $\beta_{A}<2$ and $C=0$. Then the real eigenvalues of (3.2) satisfy

$$
\min \left\{\alpha_{A}, \frac{\alpha_{S}}{\omega \beta_{A}}\right\} \leq \lambda \leq \max \left\{\beta_{A}, \frac{\beta_{S}\left(2-\alpha_{A}^{R}\right)}{\omega}\right\} .
$$

And if $\lambda_{I} \neq 0$, then the complex eigenvalues of (3.2) satisfy

$$
\frac{\omega \alpha_{A}+\alpha_{S}\left(2-\beta_{A}^{R}\right)}{2 \omega} \leq \lambda_{R} \leq \frac{\omega \beta_{A}+\beta_{S}\left(2-\alpha_{A}^{R}\right)}{2 \omega},\left|\lambda_{I}\right| \leq \sqrt{\frac{\beta_{S}}{\omega}} \max \left\{1-\alpha_{A}^{R},\left|\beta_{A}^{R}-1\right|\right\} .
$$

To develop eigenvalue bounds for RMCP we will use Theorem 3.2, and particularly the results regarding the real eigenvalues of $\mathcal{M}_{\omega}^{-1} \mathcal{A}$. The following theorem gives very simple estimates of the eigenvalues of the RMCP preconditioned matrix in terms of $\omega$. 
3.3. Theorem. Let $1 \leq \beta_{A}^{R} \leq \beta_{A}<2$.

For $\alpha_{A}^{R}<1$, any real eigenvalue $\lambda$ of $\mathcal{M}_{\omega}^{-1} \mathcal{A}$ satisfies

$$
\min \left\{\alpha_{A}, \frac{\alpha_{S}}{2 \omega}\right\} \leq \lambda \leq \max \left\{\beta_{A}, \frac{2 \beta_{S}}{\omega}\right\} .
$$

Moreover, the complex eigenvalues $\lambda$ of $\mathcal{M}_{\omega}^{-1} \mathcal{A}$ satisfy

$$
\frac{\alpha_{A}}{2} \leq \lambda_{R} \leq \frac{\beta_{A}}{2}+\frac{2 \beta_{S}-\alpha_{C}}{2 \omega},\left|\lambda_{I}\right| \leq \sqrt{\frac{\beta_{S}}{\omega}} .
$$

For $\alpha_{A}^{R} \geq 1$, any real eigenvalue $\lambda$ of $\mathcal{M}_{\omega}^{-1} \mathcal{A}$ satisfies

$$
\min \left\{\alpha_{A}, \frac{\alpha_{S}}{2 \omega}\right\} \leq \max \left\{\beta_{A}, \frac{2\left(\beta_{S}+\beta_{C}\right)}{\omega}\right\} .
$$

Moreover, the complex eigenvalues $\lambda$ of $\mathcal{M}_{\omega}^{-1} \mathcal{A}$ satisfy

$$
\frac{\alpha_{A}}{2} \leq \lambda_{R} \leq \frac{\beta_{A}}{2}+\frac{\beta_{S}+\beta_{C}}{\omega},\left|\lambda_{I}\right| \leq \sqrt{\frac{\beta_{S}}{\omega}}\left(\beta_{A}^{R}-1\right) .
$$

Proof. For $\alpha_{A}^{R}<1$, from (3.3) we have

$$
\min \left\{\alpha_{A}, \frac{\alpha_{S}+\alpha_{C}\left(\beta_{A}-1\right)}{\omega \beta_{A}}\right\} \leq \lambda \leq \max \left\{\beta_{A}, \frac{\beta_{S}\left(2-\alpha_{A}^{R}\right)-\alpha_{C}\left(1-\alpha_{A}^{R}\right)}{\omega}\right\} .
$$

Using $\alpha_{C} \geq 0,1<\beta_{A}<2$ and $\alpha_{A}^{R}<1$, we have

$$
\min \left\{\alpha_{A}, \frac{\alpha_{S}}{2 \omega}\right\} \leq \lambda \leq \max \left\{\beta_{A}, \frac{2 \beta_{S}}{\omega}\right\} .
$$

Using $1<\beta_{A}^{R}<2$ and $\alpha_{A}^{R}<1$, from (3.4) and (3.5) we have

and

$$
\begin{aligned}
\frac{\alpha_{A}}{2} \leq \lambda_{R} & \leq \frac{\omega \beta_{A}+\beta_{S}\left(2-\alpha_{A}^{R}\right)-\alpha_{C}\left(1-\alpha_{A}^{R}\right)}{2 \omega} \\
& =\frac{\beta_{A}}{2}+\frac{\beta_{S}+\left(\beta_{S}-\alpha_{C}\right)\left(1-\alpha_{A}^{R}\right)}{2 \omega} \\
& \leq \frac{\beta_{A}}{2}+\frac{2 \beta_{S}-\alpha_{C}}{2 \omega}
\end{aligned}
$$

$$
\left|\lambda_{I}\right| \leq \sqrt{\frac{\beta_{S}}{\omega}}\left(\beta_{A}^{R}-1\right) .
$$

For $\alpha_{A}^{R} \geq 1$, from (3.6) we have

$$
\min \left\{\alpha_{A}, \frac{\alpha_{S}+\alpha_{C}\left(\beta_{A}-1\right)}{\omega \beta_{A}}\right\} \leq \lambda \leq \max \left\{\beta_{A}, \frac{\beta_{S}\left(2-\alpha_{A}^{R}\right)+\beta_{C}\left(\alpha_{A}^{R}-1\right)}{\omega}\right\} .
$$

Using $\alpha_{C} \geq 0,1<\beta_{A}<2$ and $\alpha_{A}^{R} \geq 1$, we have

$$
\min \left\{\alpha_{A}, \frac{\alpha_{S}}{2 \omega}\right\} \leq \lambda \leq \max \left\{\beta_{A}, \frac{2 \beta_{S}+\beta_{C} \alpha_{A}^{R}}{\omega}\right\} \leq \max \left\{\beta_{A}, \frac{2\left(\beta_{S}+\beta_{C}\right)}{\omega}\right\} .
$$

Using $1<\beta_{A}^{R}<2$ and $\alpha_{A}^{R} \geq 1$, from (3.7) and (3.8) we have

$$
\begin{aligned}
\frac{\alpha_{A}}{2} \leq \lambda_{R} & \leq \frac{\beta_{A}}{2}+\frac{2 \beta_{S}+\alpha_{A}^{R} \beta_{C}}{2 \omega} \leq \frac{\beta_{A}}{2}+\frac{\beta_{S}+\beta_{C}}{\omega}, \\
\left|\lambda_{I}\right| & \leq \sqrt{\frac{\beta_{S}}{\omega}}\left(\beta_{A}^{R}-1\right) .
\end{aligned}
$$

Remark 3.2 Theorem 2 in [37] also gives very simple estimates of the eigenvalues of the RMCP preconditioned matrix in terms of $\omega$, but this result in Theorem 2 is not generally true. In fact, by investigating the proof of Theorem 2, the bound of the 
eigenvalue $\lambda$ of the preconditioned matrix $\mathcal{M}_{\omega}^{-1} \mathcal{A}$ not only depends on whether $\alpha_{A}^{R}$ is smaller or larger than 1 , but depends on whether $\beta_{A}^{R}$ is smaller or larger than 1 . However in [37] it is not specify whether $\alpha_{A}^{R}<1$ or $\alpha_{A}^{R}>1$. It is only stated that $0<\alpha_{A} \leq \alpha_{A}^{R}$ from $\left[\alpha_{A}^{R}, \beta_{A}^{R}\right] \subset\left[\alpha_{A}, \beta_{A}\right]$. Similarly, the conditions in Theorem 2 [37] do not also specify whether $\beta_{A}^{R}<1$ or $\beta_{A}^{R}>1$. It is only stated that $\beta_{A}^{R} \leq \beta_{A}$ from $\left[\alpha_{A}^{R}, \beta_{A}^{R}\right] \subset\left[\alpha_{A}, \beta_{A}\right]$. In this case, Theorem 3.3 perfects the results in Theorem 2 in [37].

Example 3.1 Let

$$
A=\left[\begin{array}{ccc}
0.5 & 0 & 0 \\
0 & 1.5 & 0 \\
0 & 0 & 1
\end{array}\right], B=\left[\begin{array}{lll}
1 & 0 & 1 \\
0 & 1 & 0
\end{array}\right], C=\left[\begin{array}{cc}
0.5 & 0 \\
0 & 0.6
\end{array}\right]
$$

For convenience, we can choose $P_{A}=I$ and $P_{S}=2 I$. Then $A_{P}=P_{A}^{-1 / 2} A P_{A}^{-1 / 2}=A$,

$$
S=B P_{A}^{-1} B^{T}+C=\left[\begin{array}{cc}
2.5 & 0 \\
0 & 1.6
\end{array}\right], S_{P}=P_{S}^{-1 / 2} S P_{S}^{-1 / 2}=\left[\begin{array}{cc}
1.25 & 0 \\
0 & 0.8
\end{array}\right]
$$

and

$$
\widehat{C}=P_{S}^{-1 / 2} C P_{S}^{-1 / 2}=\left[\begin{array}{cc}
0.25 & 0 \\
0 & 0.3
\end{array}\right]
$$

Therefore, $\alpha_{A}=\lambda_{\min }\left(A_{P}\right)=0.5, \beta_{A}=\lambda_{\max }\left(A_{P}\right)=1.5, \alpha_{S}=\lambda_{\min }\left(S_{P}\right)=0.8, \beta_{S}=$ $\lambda_{\max }\left(S_{P}\right)=1.25, \alpha_{C}=\lambda_{\min }(\widehat{C})=0.25$ and $\beta_{C}=\lambda_{\max }(\widehat{C})=0.3$.

Since $R=P_{S}^{-1 / 2} B P_{A}^{-1 / 2}=P_{S}^{-1 / 2} B$,

$$
A_{R}=\left(R R^{T}\right)^{-1} R A_{P} R^{T}=\left[\begin{array}{cc}
0.75 & 0 \\
0 & 1.5
\end{array}\right] \text {, }
$$

This shows that $\alpha_{A}^{R}=\lambda_{\min }\left(A_{R}\right)=0.75, \beta_{A}^{R}=\lambda_{\max }\left(A_{R}\right)=1.5<2$.

If $\omega=2$, all the eigenvalues of $\mathcal{M}_{\omega}^{-1} \mathcal{A}$ are $\lambda\left(\mathcal{M}_{\omega}^{-1} \mathcal{A}\right)=\{0.3283,1,1.4467,0.6250 \pm$ $0.2165 i$ \}. From Theorem 3.3, any real eigenvalue $\lambda$ of $\mathcal{M}_{\omega}^{-1} \mathcal{A}$ satisfies

$$
0.2<\lambda<1.5
$$

and the complex eigenvalue $\lambda$ of $\mathcal{M}_{\omega}^{-1} \mathcal{A}$ satisfies

$$
0.25<\lambda_{R}<1.3125,\left|\lambda_{I}\right|<0.7906 .
$$

Obviously, $0.3283,1$ and 1.4467 lie in $(0.2,1.5), 0.6250 \in(0.25,1.3125)$ and $| \pm 0.2165|<$ 0.7906 .

Based on Theorem 2 in [37], any real eigenvalue $\lambda$ of $\mathcal{M}_{\omega}^{-1} \mathcal{A}$ satisfies

$$
0.5<\lambda<6,
$$

and the complex eigenvalue $\lambda$ of $\mathcal{M}_{\omega}^{-1} \mathcal{A}$ satisfies

$$
0.25<\lambda_{R}<2.75,\left|\lambda_{I}\right|<1.5811 \text {. }
$$

Obviously, $0.3283 \notin(0.5,6), 0.6250 \in(0.25,2.75)$ and $| \pm 0.2165|<1.5811$.

If $\omega=\frac{1}{2}$, all the eigenvalues of $\mathcal{M}_{\omega}^{-1} \mathcal{A}$ are $\lambda\left(\mathcal{M}_{\omega}^{-1} \mathcal{A}\right)=\{0.6044,1,2.8956,1.3 \pm 0.4583 i\}$. From Theorem 3.3, any real eigenvalue $\lambda$ of $\mathcal{M}_{\omega}^{-1} \mathcal{A}$ satisfies

$$
0.5<\lambda<5
$$

and the complex eigenvalue $\lambda$ of $\mathcal{M}_{\omega}^{-1} \mathcal{A}$ satisfies

$$
0.25<\lambda_{R}<3,\left|\lambda_{I}\right|<1.5811
$$

Obviously, $0.6044,1,2.8956$ lie in $(0.5,5), 1.3 \in(0.25,3)$ and $| \pm 0.4583|<1.5811$.

Based on Theorem 2 in [37], any real eigenvalue $\lambda$ of $\mathcal{M}_{\omega}^{-1} \mathcal{A}$ satisfies

$$
0.2<\lambda<1.5
$$


and the complex eigenvalue $\lambda$ of $\mathcal{M}_{\omega}^{-1} \mathcal{A}$ satisfies

$$
0.25<\lambda_{R}<1.8125,\left|\lambda_{I}\right|<0.7906 .
$$

Obviously, $2.8956 \notin(0.2,1.5), 1.3 \in(0.25,1.8125)$ and $| \pm 0.4583|<0.7906$.

Example 3.2 Let

$$
A=\left[\begin{array}{ccc}
0.5 & 0 & 0 \\
0 & 1.5 & 0 \\
0 & 0 & 0.25
\end{array}\right], B=\left[\begin{array}{ccc}
-1 & 0 & -1 \\
0 & 1 & 0
\end{array}\right], C=\left[\begin{array}{cc}
0 & 0 \\
0 & 0.2
\end{array}\right]
$$

For convenience, we can choose $P_{A}=I$ and $P_{S}=\frac{5}{3} I$. Then $A_{P}=P_{A}^{-1 / 2} A P_{A}^{-1 / 2}=A$,

$$
S=B P_{A}^{-1} B^{T}+C=\left[\begin{array}{cc}
2 & 0 \\
0 & 1.2
\end{array}\right], S_{P}=P_{S}^{-1 / 2} S P_{S}^{-1 / 2}=\left[\begin{array}{cc}
1.2 & 0 \\
0 & 0.72
\end{array}\right]
$$

and

$$
\widehat{C}=P_{S}^{-1 / 2} C P_{S}^{-1 / 2}=\left[\begin{array}{cc}
0 & 0 \\
0 & 0.12
\end{array}\right] .
$$

Therefore, $\alpha_{A}=\lambda_{\min }\left(A_{P}\right)=0.25, \beta_{A}=\lambda_{\max }\left(A_{P}\right)=1.5, \alpha_{S}=\lambda_{\min }\left(S_{P}\right)=0.72$, $\beta_{S}=\lambda_{\max }\left(S_{P}\right)=1.2, \alpha_{C}=\lambda_{\min }(\widehat{C})=0$ and $\beta_{C}=\lambda_{\max }(\widehat{C})=0.12$.

Since $R=P_{S}^{-1 / 2} B P_{A}^{-1 / 2}=P_{S}^{-1 / 2} B$,

$$
A_{R}=\left(R R^{T}\right)^{-1} R A_{P} R^{T}=\left[\begin{array}{cc}
0.375 & 0 \\
0 & 1.5
\end{array}\right],
$$

This shows that $\alpha_{A}^{R}=\lambda_{\min }\left(A_{R}\right)=0.375, \beta_{A}^{R}=\lambda_{\max }\left(A_{R}\right)=1.5<2$.

If $\omega=3$, all the eigenvalues of $\mathcal{M}_{\omega}^{-1} \mathcal{A}$ are $\lambda\left(\mathcal{M}_{\omega}^{-1} \mathcal{A}\right)=\{0.2147,0.3927,1.4533,0.5687 \pm$ $0.3675 i$ \}. From Theorem 3.3, any real eigenvalue $\lambda$ of $\mathcal{M}_{\omega}^{-1} \mathcal{A}$ satisfies

$$
0.12<\lambda<1.5
$$

and the complex eigenvalue $\lambda$ of $\mathcal{M}_{\omega}^{-1} \mathcal{A}$ satisfies

$$
0.125<\lambda_{R}<1.15,\left|\lambda_{I}\right|<0.6325 \text {. }
$$

Obviously, $0.2147,0.3927$ and 1.4533 lie in $(0.12,1.5), 0.5687 \in(0.125,1.15)$ and $\mid \pm$ $0.3675 \mid<0.6325$.

Based on Theorem 2 in [37], any real eigenvalue $\lambda$ of $\mathcal{M}_{\omega}^{-1} \mathcal{A}$ satisfies

$$
0.25<\lambda<7.2
$$

and the complex eigenvalue $\lambda$ of $\mathcal{M}_{\omega}^{-1} \mathcal{A}$ satisfies

$$
0.125<\lambda_{R}<3.3,\left|\lambda_{I}\right|<1.8974 \text {. }
$$

Obviously, $0.2147 \notin(0.25,7.2), 0.5687 \in(0.125,3.3)$ and $| \pm 0.3675|<1.8974$.

If $\omega=\frac{1}{3}$, all the eigenvalues of $\mathcal{M}_{\omega}^{-1} \mathcal{A}$ are $\lambda\left(\mathcal{M}_{\omega}^{-1} \mathcal{A}\right)=\{0.3649,0.6642,5.571,1.38 \pm$ $0.66 i\}$. From Theorem 3.3, any real eigenvalue $\lambda$ of $\mathcal{M}_{\omega}^{-1} \mathcal{A}$ satisfies

$$
0.25<\lambda<7.2
$$

and the complex eigenvalue $\lambda$ of $\mathcal{M}_{\omega}^{-1} \mathcal{A}$ satisfies

$$
0.125<\lambda_{R}<4.35,\left|\lambda_{I}\right|<1.8974 \text {. }
$$

Obviously, $0.3649,0.6642$ and 5.571 lie in $(0.25,7.2), 1.38 \in(0.125,4.35)$ and $| \pm 0.66|<$ 1.8974.

Based on Theorem 2 in [37], any real eigenvalue $\lambda$ of $\mathcal{M}_{\omega}^{-1} \mathcal{A}$ satisfies

$$
0.12<\lambda<1.5
$$

and the complex eigenvalue $\lambda$ of $\mathcal{M}_{\omega}^{-1} \mathcal{A}$ satisfies

$$
0.125<\lambda_{R}<1.7,\left|\lambda_{I}\right|<0.6325 .
$$


Obviously, $5.571 \notin(0.12,1.5), 1.38 \in(0.125,1.7)$ and $| \pm 0.66| \nless 0.6325$.

Numerical results of Examples 3.1 and 3.2 show that the eigenvalue distribution of the preconditioned matrix $\mathcal{M}_{\omega}^{-1} \mathcal{A}$ in Theorem 3.3 is more tighter than that of Theorem 2 in [37]. This shows that Theorem 3.3 provide valid bounds for all the real eigenvalues of the preconditioned matrix $\mathcal{M}_{\omega}^{-1} \mathcal{A}$ and also provide valid bounds for the real and imaginary parts of all the complex eigenvalues of the preconditioned matrix $\mathcal{M}_{\omega}^{-1} \mathcal{A}$.

\section{Conclusion}

In this paper, our goal is to discuss the eigenvalue distribution of a family of relaxed mixed constraint preconditioner (RMCP) for saddle point problems. Some valid bounds for all the eigenvalues of the corresponding preconditioned matrix are obtained and some corresponding theoretical results in $[36,37,44]$ have been improved. With regard to the application of RMCP, one can see [34,36,37] for more details.

\section{Acknowledgements}

The authors would like to thank the anonymous referee and Editor Yücel Turaş for providing helpful suggestions, which greatly improved the paper. This work was supported by Natural Science Foundation of China (No.11301009), No.17HASTIT012, Natural Science Foundations of Henan Province (No.15A110007) and Project of Young Core Instructor of Universities in Henan Province (No. 2015GGJS-003).

\section{References}

[1] Z.-Z. Bai, M.K. Ng, Z.-Q. Wang, Constraint preconditioners for symmetric indefinite matrices, SIAM J. Matrix Anal. Appl., 31 (2009) 410-433.

[2] Z.-Z. Bai, B.N. Parlett, Z.-Q. Wang, On generalized successive overrelaxation methods for augmented linear systems, Numer. Math., 102 (2005) 1-38.

[3] Z.-Z. Bai, G.H. Golub, M.K. Ng, Hermitian and skew-Hermitian splitting methods for nonHermitian positive definite linear systems, SIAM J. Matrix Anal. Appl., 24 (2003) 603-626.

[4] Z.-Z. Bai, G.H. Golub, J.-Y. Pan, Preconditioned Hermitian and skew-Hermitian splitting methods for non-Hermitian positive semidefinite linear systems, Numer. Math., 98 (2004) $1-32$.

[5] Z.-Z. Bai, G.H. Golub, Accelerated Hermitian and skew-Hermitian splitting iteration methods for saddle-point problems, IMA J. Numer. Anal., 27 (2007) 1-23.

[6] Z.-Z. Bai, G.H. Golub, C.-K. Li, Convergence properties of preconditioned Hermitian and skew-Hermitian splitting methods for non-Hermitian positive semidefinite matrices, Math. Comput., 76 (2007) 287-298.

[7] Z.-Z. Bai, Optimal parameters in the HSS-like methods for saddle-point problems, Numer. Linear Algebra Appl., 16 (2009) 447-479.

[8] Z.-Z. Bai, M.K. Ng, On inexact preconditioners for nonsymmetric matrices, SIAM J. Sci. Comput., 26 (2005) 1710-1724.

[9] Z.-Z. Bai, Structured preconditioners for nonsingular matrices of block two-by-two structures, Math. Comput., 75 (2006) 791-815.

[10] M. Benzi, G.H. Golub, J. Liesen, Numerical solution of saddle point problems, Acta Numer., $14(2005)$ 1-137.

[11] M. Benzi, A generalization of the Hermitian and skew-Hermitian splitting iteration, SIAM J. Matrix Anal. Appl., 31 (2009) 360-374.

[12] M. Benzi, G.H. Golub, A preconditioner for generalized saddle point problems, SIAM J. Matrix Anal. Appl., 26 (2004) 20-41.

[13] M. Benzi, M. Ng, Q. Niu, Z. Wang, A relaxed dimensional factorization preconditioner for the incompressible Navier-Stokes equations, J. Comput. Phys., 230 (2011) 6185-6202.

[14] M. Benzi, X.-P. Guo, A dimensional split preconditioner for Stokes and linearized NavierStokes equations, Appl. Numer. Math., 61 (2011) 66-76. 
[15] M. Benzi, M.A. Olshanskii, An augmented Lagrangian-based approach to the Oseen problem, SIAM J. Sci. Comput., 28 (2006) 2095-2113.

[16] M. Benzi, M.A. Olshanskii, Z. Wang, Modified augmented Lagrangian preconditioners for the incompressible Navier-Stokes equations, Int. J. Numer. Methods Fluids, 66 (2011) 486508.

[17] M. Benzi, V. Simoncini, On the eigenvalues of a class of saddle point matrices, Numer. Math., 103 (2006) 173-196.

[18] R. Bridson, KKTDirect: a direct solver package for saddle-point (KKT) matrices, Preprint, Department of Computer Science, University of British Columbia, 2009.

[19] A.C. de Niet, F.W. Wubs, Numerically stable $L D L^{T}$-factorization of $F$-type saddle point matrices, IMA J. Numer. Anal., 29 (2009) 208-234.

[20] Z.-H. Cao, Augmentation block preconditioners for saddle point-type matrices with singular $(1,1)$ blocks, Numer. Linear Algebra Appl., 15 (2008) 515-533.

[21] Z.-H. Cao, A note on block diagonal and constraint preconditioners for non-symmetric indefinite linear systems, Int. J. Comput. Math., 83 (2006) 383-395.

[22] D.J. Silvester, A.J. Wathen, Fast iterative solution of stabilized Stokes systems, Part II: Using general block preconditioners, SIAM J. Numer. Anal., 31 (1994) 1352-1367.

[23] I. Perugia, V. Simoncini, Block-diagonal and indefinite symmetric preconditioners for mixed finite element formulations, Numer. Linear Algebra Appl., 7 (2000) 585-616.

[24] V. Simoncini, Block triangular preconditioners for symmetric saddle-point problems, Appl. Numer. Math., 49 (2004) 63-80.

[25] Z.-H. Cao, Positive stable block triangular preconditioners for symmetric saddle point problems, Appl. Numer. Math., 57 (2007) 899-910.

[26] Z.-H. Cao, Block triangular Schur complement preconditioners for saddle piont problems and application to the Oseen equation, Appl. Numer. Math., 60 (2010) 193-207.

[27] Z.-H. Cao, A note on constraint preconditioning for nonsymmetric indefinite matrices, SIAM J. Matrix Anal. Appl., 24 (2002) 121-125.

[28] A. Klawonn, Block-triangluar preconditioners for saddle point problems with a penalty term, SIAM J. Sci. Comput., 19 (1998) 172-184

[29] M.F. Murphy, G.H. Golub, A.J. Wathen, A note on preconditoning for indefinite linear systems, SIAM J. Sci. Comput., 21 (2000) 1969-1972.

[30] I.C.F. Ipsen, A note on preconditioning nonsymmetric matrices, SIAM J. Sci. Comput., 23 (2001) 1050-1051.

[31] C. Keller, N.I.M. Gould, A.J. Wathen, Constraint preconditioning for indefinite linear systems, SIAM J. Matrix Anal. Appl., 21 (2000) 1300-1317.

[32] H.S. Dollar, Constraint-style preconditioners for regularized saddle point problems, SIAM J. Matrix Anal. Appl., 29 (2007) 672-684.

[33] S.-L. Wu, T.-Z. Huang, X.-L. Zhao, A modified SSOR iterative method for augmented systems, J. Comput. Appl. Math., 228 (2009) 424-433.

[34] L. Bergamaschi, M. Ferronato, G. Gambolati, Mixed constraint preconditioners for the iterative solution of FE coupled consolidation equations, J. Comput. Phys., 227 (2008) 98859897.

[35] L. Bergamaschi, M. Ferronato, G. Gambolati, Performance and robustness of block constraint preconditioners in finite element coupled consolidation problems, Int. J. Numer. Methods Engrg., 81 (2010) 381-402.

[36] L. Bergamaschi, On eigenvalue distribution of constraint-preconditioned symmetric saddle point matrices, Numer. Linear Algebra Appl., 19 (2012) 754-772.

[37] L. Bergamaschi, A. Martínez, RMCP: Relaxed Mixed Constraint Preconditioners for saddle point linear systems arising in geomechanics, Comput. Methods Appl. Mech. Engrg., 221-222 (2012) 54-62.

[38] L. Bergamaschi, M. Ferronato, G. Gambolati, Novel preconditioners for the iterative solution to FE-discretized coupled consolidation equations, Comput. Methods Appl. Mech. Engrg., 196 (2007) 2647-2656.

[39] S.-L. Wu, T.-Z. Huang, C.-X. Li, Modified block preconditioners for the discretized timeharmonic Maxwell equations in mixed form, J. Comput. Appl. Math., 237 (2013) 419-431. 
[40] C.-X. Li, S.-L. Wu, An improved lower bound on a positive stable block triangular preconditioner for saddle point problems, Appl. Math. Lett., 25 (2012) 2174-2178.

[41] S.-L. Wu, C.-X. Li, A note on parameterized block triangular preconditioners for generalized saddle point problems, Appl. Math. Comput., 219 (2013) 7907-7916.

[42] S.-L. Wu, T.-Z. Huang, C.-X. Li, Block triangular preconditioner for static Maxwell equations, Comput. Appl. Math., 30 (2011) 589-612.

[43] S.-L. Wu, C.-X. Li, Indefinite block triangular preconditioner for symmetric saddle point problems, Calcolo, 50 (2013) 1-15.

[44] S.-L. Wu, L. Bergamaschi, C.-X. Li, A note on eigenvalue distribution of constraintpreconditioned symmetric saddle point matrices, Numer. Linear Algebra Appl., 21 (2014) 171-174. 